\title{
Monte Carlo simulations of krypton adsorption in nanopores: Influence of pore wall heterogeneity on the adsorption mechanism
}

\author{
Bogdan Kuchta, Philip Llewellyn, and Renaud Denoyel \\ Laboratoire des Materiaux Divises, Revetement, Electroceramiques, Universite de Provence, \\ Centre de Saint-Jerome, 13397 Marseille Cedex 20, France \\ E-mail: kuchta@up.univ-mrs.fr \\ Lucyna Firlej \\ Groupe de Dynamique des Phases Condensees \\ Universite Montpellier II, 34095, Montpellier Cedex 5, France
}

\begin{abstract}
We present molecular simulation results of the adsorption of krypton in a model of MCM-41 mesoporous material. The adsorption isotherm and adsorption enthalpies have been studied at $77 \mathrm{~K}$. The comparison of experimental and simulation data allows us to analyze how the available interaction models ( $\mathrm{Kr}-\mathrm{Kr}$ and $\mathrm{Kr}-$ walls) are able to reproduce the experimental situation. The role of the heterogeneous interactions versus homogenous model is studied and compared with the previous simulation results of nitrogen adsorption in MCM-41. The results show that a model of ideal cylindrical pores gives qualitatively and quantitatively different results. A distribution of the adsorption sites must exist to explain the loading at low pressure (below capillary condensation). Such distribution in MCM-41 is a consequence of non-homogenous walls that contain a wide variety of attractive sites ranging from weakly attractive silica-type to highly attractive regions. In our simulations, the MCM-41 structure is modeled as an amorphous array of oxygen and silicon atoms, each one interacting with an adsorptive atom via the atom-atom potential. The distribution of the adsorption sites is merely a consequence of local atomic structure. Such a model of the wall reproduces the smooth increase in loading seen experimentally.
\end{abstract}

PACS: 64.70.Fx, 68.43.De, 68.43.Fg, 61.43.Bn

\section{Introduction}

An application of computer modeling methods to study adsorption in pores is strongly dependent on the interaction model, in particular, the interaction of the adsorptive with the walls of the adsorbent. Much of the work was performed using simple models with regular shaped pores and smooth walls. However, there are relatively few real adsorbents that conform exactly to either of regular (cylindrical and slit) shapes. A strong influence of the pore geometry on the thermodynamics of systems in confined geometry is well known [1]. It is obvious that the discrete structure of walls must play a role in the adsorption mechanism, especially in ultra-micropores where the average diameter is few times the size of the adsorbate.

MCM-41 material, synthesized in 1992, is a model nano-porous material with narrow pore distribution (with mean diameter in the range 2-10 $\mathrm{nm}$ ) and straight unconnected channels. These features are of particular interest because they make it an ideal model as well as practical adsorbent. Adsorption isotherms have been measured for many different adsorptives [1]. They are generally of type IV in the IUPAC classification [2], showing the strong affinity of the fluid for the pore at low pressure, followed by a gradual increase in amount adsorbed up to a sharp capillary condensation. Except for a few cases, the desorption branch of the isotherm follows a different path from the adsorption branch.

Therefore, the MCM-41 samples are very suited to analyze the capillary condensation phenomenon and to determine the phase diagram of the confined capillary phase. However, the mechanism of the adsorption depends strongly on the detailed structure of walls on 
the atomic level. The amorphous structure of the pore material produces a distribution of adsorption sites and makes non-homogeneous the interaction with the wall. The recent simulations of nitrogen in the MCM-41 pores [1] have shown that the only way to reproduce the smooth increase in loading seen experimentally is to use a model with a heterogeneous surface.

In the present paper, we model the krypton adsorption in nanopores. Krypton is an example of capillary solidification at temperatures where the sorption isotherm can be measured [3,4]. In this paper we carry out an analysis of the influence of the krypton-wall interaction on the phenomena. It is the first step to understand the mechanism of the krypton adsorption in MCM-41 material. We analyze the influence of the random distribution of the adsorption sites on the adsorption isotherms. The source of the heterogeneity and adsorption sites distribution is the atomic matrix disorder of the amorphous walls of the MCM-41 material.

\section{The interaction model and the simulation methodology}

The MCM-41 structure is modeled as a disordered array of oxygen and silicon atoms, each one interacting with an adsorptive atom via the potential based on the model proposed by Pellenq et al. [5]. The skeletal density is assumed to be the same as that proposed by Maddox et al. [1], being of $27 T$-sites ( $\mathrm{Si}$ atoms) per $\mathrm{nm}^{3}$ for the solid MCM-41 material. Having this decided, we created disordered pseudo-hexagonal $T$-site bulk structure where we randomly inserted oxygen atoms with a constraint that the $\mathrm{Si}-\mathrm{O}$ distance $d=$ $=1.61 \AA$. Then, we formed the pores by removing all atoms within assumed pore diameter $D$ and wall thickness $W$. The simulation results presented in this paper correspond to a single pore of $D=40 \AA$ and $W=10 \AA$. We have not included inter-pore interaction because the fluid-fluid interaction between atoms in adjacent pores proved to be negligibly small for wall thickness of $10 \AA$.

The $\mathrm{Kr}-\mathrm{Kr}$ and $\mathrm{Kr}-$ wall $(\mathrm{Kr}-\mathrm{O}, \mathrm{Kr}-\mathrm{Si})$ interactions include dispersion and repulsive short-range contributions. No induction energy has been used. The model based directly on the parameters taken from Pellenq and Levitz [5] turned out to give the $\mathrm{Kr}-$ wall energies much smaller than the values reported experimentally. This fact was reflected in the adsorption behavior, which was more like of type III in the IUPAC classification [2]. Therefore, in order to reproduce the experimental enthalpies of adsorption, we have increased 2 times the strength of the interaction. Such model has given reasonable agreement with experi- mental measurement, although additional «engineering» of the interaction parameters will be needed to reproduce $\mathrm{Kr}$ in MCM-41 material.

The wall surface roughness is taken into account. We constructed a 3D grid in the Monte Carlo (MC) box (a pore of the diameter $D=40 \AA$, with axial length $L=25 \AA$, and periodic boundary conditions). The interaction energy at each grid point has been precalculated and the linear interpolation was used during the simulation runs to calculate the off-grid energies. Our model of the MCM-41 mesopore produces a distribution of the adsorption sites strongly peaked around $E_{\min }=-1000 \mathrm{~K}$ with the half-width equal to 500-700 K. This feature seems to have an important impact on the adsorption mechanism as is illustrated below. However, currently we do not have any knowledge of experimental data to compare with our theoretical distribution.

The conventional grand canonical MC ensemble has been applied. So, the simulation box is assumed to be in equilibrium with the bulk gas, which obeys the ideal gas law. Trial moves include translations of atoms, insertion of new atoms and removal of existing ones. Typical runs contained the minimum number of MC steps of the order of $10^{6}$ (per atom). The main results are extracted from the previously equilibrated runs. The equilibration runs are giving information concerning the kinetics of the transformations observed in the system.

\section{Krypton adsorption at $77 \mathrm{~K}$}

Our first task was to compare the adsorption of krypton in ideal cylindrical and corrugated pores. Figure 1 shows the simulated adsorption isotherm in the corrugated pore. It reproduces the experimental data in a reasonable way. However, the slope of the simu-

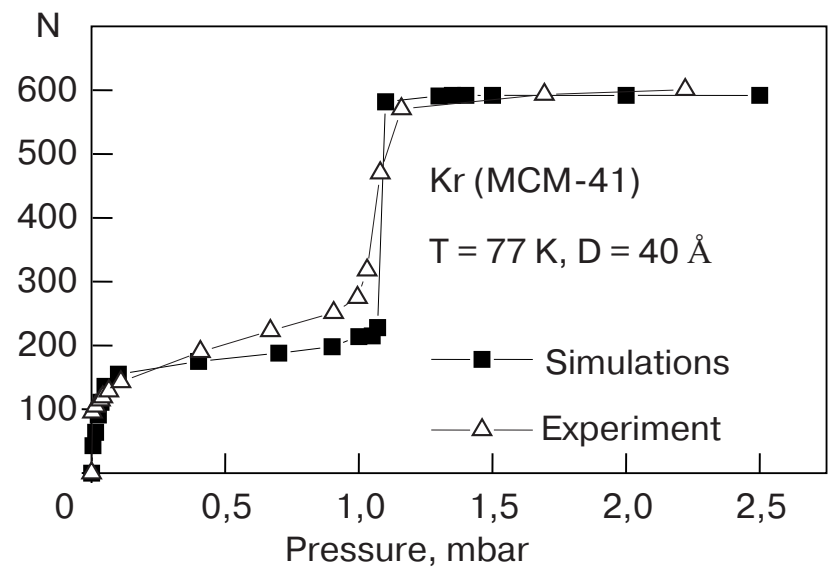

Fig. 1. Experimental and simulated adsorption isotherms. The amount adsorbed is given in units of number of atoms per volume of the Monte Carlo box. 


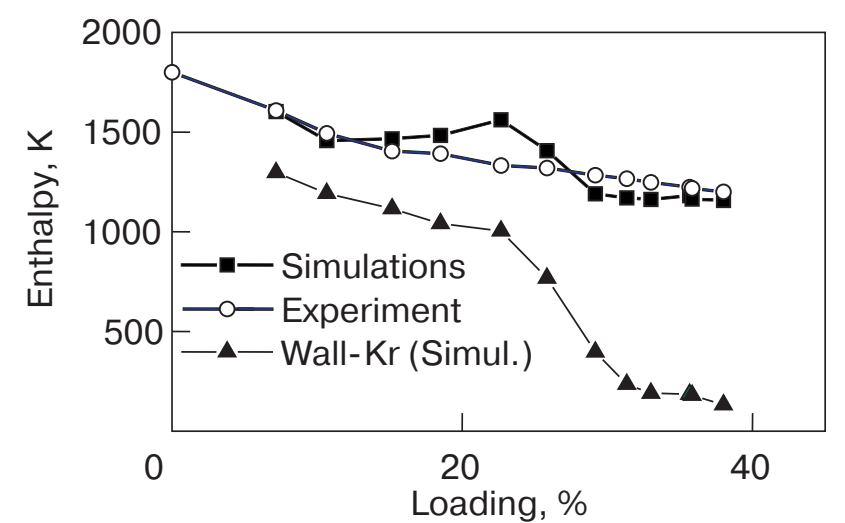

Fig. 2. Enthalpies of a adsorption as function of loading. The numerical results show the total enthalpy and, separately, the contribution from the atom-wall interaction.

lated isotherm is more flat than the experimental one below the pressure of the capillary condensation and the capillary condensation occurs much more rapidly. The latter difference is usually explained by a distribution of the pore size in the real material that is not present in the model. The former one mirrors the differences in the $\mathrm{Kr}-$ wall interaction, in particular, in the distribution and energies of the adsorption sites on the MCM-41 walls. The adsorption isotherm calculated for the ideal cylindrical pore (that is with $\delta$-like adsorption site distribution) is totally flat below the pore condensation pressure. The pressure itself is shifted towards lower values.

The adsorption enthalpy as a function of the loading gives additional insight into this problem (see Fig. 2). Experimental data show energy uniformly decreasing in the range from 0 to $40 \%$ of the maximal loading. The calculated curve, however, shows a local maximum at about $25 \%$ of the loading. This is a consequence of our wall structure and the distribution of the adsorption sites as discussed above. The $\mathrm{Kr}-$ wall contribution to the total enthalpy (Fig. 2) shows different slopes above and below the energy $E \simeq$ $\simeq-1000 \mathrm{~K}$. This value corresponds to the maximum of adsorption site distribution.

The influence of the strong corrugation seems to have an effect on the microscopic states in equilibrium and on the mechanism of the transformation. During the simulation runs we have observed different metastable configurations that existed as intermediate states. As an example we present the simulated fluctuations of the number of atoms in the MC box when the system is undergoing the capillary condensation (Fig. 3). It clearly shows an intermediate, metastable state, before the pore condensation is completed. It represents a formation of the second layer, which ex-

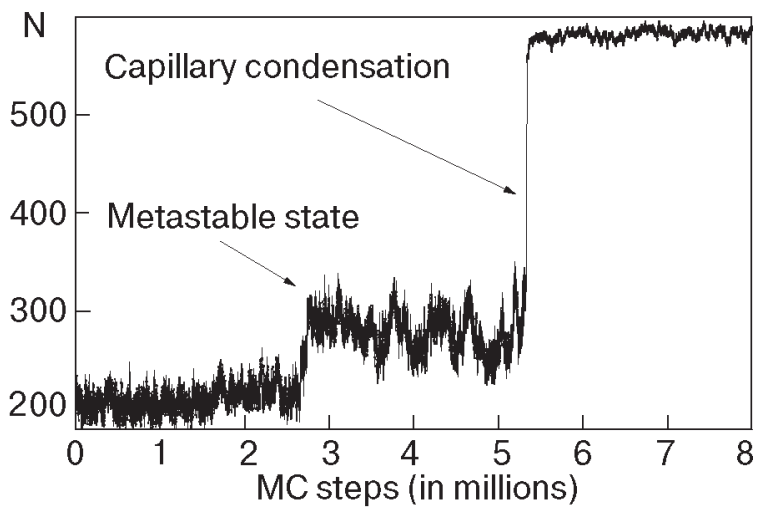

Fig. 3. The fluctuations of the number of atoms in the simulation box as a function of the Monte Carlo steps, at the capillary condensation pressure.

ists only within limited time of simulation. Instead of stabilizing it, the system undergoes a transformation into the condensed phase.

\section{Conclusions}

The results presented here show the profound influence of a corrugated wall structure on the mechanism of adsorption. The presented example shows that it could affect the profile of an adsorption isotherm and the enthalpy of adsorption. This influence is particularly important in the range of pressures below the capillary condensation. The slope of the adsorption isotherm is determined by the distribution of the energies of the adsorption sites. This result is compatible with the conclusion reported by Maddox et al. [1] where a heterogeneous interaction model was used to reproduce the experimental form of the adsorption isotherm of nitrogen in MCM-41 system.

The existence of metastable states along the adsorption isotherm is understood and observed in many systems. The hysteresis of the adsorption-desorption curve, observed experimentally, is the most evident proof. However, our simulation shows that the nonhomogeneous wall structure induces additional, short-lived metastable states that may affect the mechanism of condensation and other phase transitions. So, we conclude that the heterogeneous interactions are responsible not only for the form of the adsorption isotherm. They also affect the mechanism of the phase transformations in the system.

The interaction model used in this paper requires additional refinement. It seems that there are no potential parameters, which describe atom-wall interaction and are transferable between different pore systems, even if they are built from the same atoms and 
similar basis units ( $\mathrm{Si}-\mathrm{O}$ bonds in our case). The most probable cause is the electron states of surface atoms modified by local imperfections of the chemical bond structure.

1. M.W. Maddox, J.P. Olivier, and K.E. Gubbins, Lagmuir 13,1737 (1997).

2. F. Rouquerol, J. Rouquerol, and K. Sing, Adsorption by Powders and Porous Solids, Academic Press, London (1999).
3. J.P. Coulomb, Y. Grillet, P. Llewellyn, C. Martin, and G. Andre, Proc. $6^{\text {th }}$ Int. Conf. Fundamen Adsorption, F. Meunier (ed.), Elsevier (1998), p. 147.

4. J.P. Coulomb, N. Floquet, Y. Grillet, P. Llewellyn, R. Kahn, and G. Andre, in: Studies in Surface Science and Catalysis, K.K. Unger (ed.), Elsevier (2000), p. 235.

5. R.J.-M. Pellenq and P.E. Levitz, Mol.Simul. (in press) 